\section{NECESIDAD DE ENSEÑANZA DE LA SALUD OCUPACIONAL EN LA EDUCACIÓN MÉDICA DE PREGRADO}

\author{
NEED FOR OCCUPATIONAL HEALTH \\ EDUCATION IN UNDERGRADUATE MEDICAL \\ EDUCATION
}

\author{
Akram Hernández-Vásquez ${ }^{1, a, b}$, \\ Fabiola Marcalaya-Benites ${ }^{2, a}$, \\ Jesús A. Santiani ${ }^{3, c}$, Luz Pretell-Paredes ${ }^{d}$
}

Sr. Editor. Los profundos cambios políticos, demográficos y económicos de los últimos años han tenido un impacto importante en la demanda y provisión de servicios de salud en el Perú, los cuales han ido adaptándose a las nuevas condiciones. Desde el año 2004 el empleo formal en el Perú viene incrementándose progresivamente, siendo los sectores agricultura, pesca y minería los que concentran la mayor cantidad de trabajadores. Este aumento nos enfrenta a nuevos desafíos en el campo de la salud de los trabajadores y de su relación con el medioambiente y, además, nos demuestra que las ciencias médicas se desenvuelven en un contexto dinámico y complejo, tanto para las instituciones como para el desempeño de los profesionales de la salud (1).

En el 2011, con la promulgación de la Ley 29783, Ley de Seguridad y Salud en el Trabajo, se configura un nuevo escenario para la salud ocupacional en el Perú al establecer su aplicación a todo sector productivo y de servicios, sea privado o público. Adicionalmente, el marco legal otorga responsabilidades a todo centro médico asistencial en lo relativo a la notificación de accidentes de trabajo y de enfermedades ocupacionales, condición que, necesariamente, requiere preparación previa de los profesionales de la salud en salud ocupacional.

La preocupación de la educación médica por la formación en salud ocupacional no debe ser solo teórica ni normativa. La Organización Mundial de la Salud ha reconocido a la salud de los trabajadores como eje fundamental para el desarrollo de las naciones, sin

\footnotetext{
1 Universidad de Chile. Chile.

Oficina de Salud Ocupacional de la Clínica Javier Prado. Lima, Perú

Hospital Guillermo Almenara Irigoyen. Lima, Perú

a Médico; ${ }^{\text {b }}$ estudiante del Magíster en Gestión y Políticas Públicas de la

Universidad de Chile, Chile; ${ }^{\mathrm{c}}$ médico neumólogo; ${ }^{\mathrm{d}}$ abogado

Recibido: 17-06-14 Aprobado: 06-08-14
}

Citar como: Hernández-Vásquez A, Marcalaya-Benites F, Santiani JA, Pretell-Paredes L. Necesidad de enseñanza de la salud ocupacional en la educación médica de pregrado. Rev Peru Med Exp Salud Publica. 2014;31(3):605-6. embargo, en muchos países de Latinoamérica, y en especial el Perú, el desarrollo de la salud ocupacional es aún incipiente y presenta, en algunos sectores, ausencia de especialistas ${ }^{(2,3)}$. Lo anterior puede verse reflejado en la poca acción por parte de las autoridades y profesionales en la intervención de personas o poblaciones que han sido afectadas por ciertas actividades productivas y/o contaminación ambiental (4). Por otro lado, la presencia de esta área de la medicina en la formación médica de pregrado en el Perú es muy escasa, lo que implica el reto de promover la formación de profesionales que sean capaces de abordar en un futuro el cuidado de sus pacientes y de los riesgos a la salud con las competencias necesarias.

En tal sentido, el desafío que nos plantea este nuevo escenario, requiere una reorientación de algunos elementos de la educación médica de pregrado, como son los planes de estudio que favorezcan el desarrollo de competencias para satisfacer y dar respuesta a las necesidades de nuestra sociedad. Sin embargo, las limitaciones de tiempo y contenido generan un tradeoff al momento del diseño del plan de estudios que termina por excluir temas que no son considerados básicos, a pesar de su importancia y reconocimiento dentro de la práctica médica, como por ejemplo, la salud ocupacional ${ }^{(5)}$. Siendo así, y teniendo en consideración que la educación de pregrado en medicina necesita evolucionar acorde al contexto actual, proponemos que los planes de estudios de pregrado incluyan a la salud ocupacional como tema integrado y transversal a cursos obligatorios donde la semiología destaque la importancia de los antecedentes ocupacionales; se estudie la fisiopatología de las enfermedades ocupacionales más frecuentes en el curso de patología; se examine la toxicología ocupacional dentro de la farmacología; que las enfermedades pulmonares, oncológicas y dermatológicas ocupacionales se profundicen en medicina interna y especialidades médicas; los aspectos preventivos y de políticas de salud de los trabajadores se circunscriban en los cursos de salud comunitaria y salud pública, y finalmente, se den a conocer los aspectos normativos en el curso de medicina legal.

Esta propuesta permitirá que los futuros médicos adquieran competencias específicas para realizar un abordaje integral y efectivo en el cuidado de la salud de los trabajadores, promoviendo así, el bienestar colectivo, la productividad y el desarrollo económico y social del país.

Fuentes de financiamiento: autofinanciado.

Conflictos de interés: los autores declaran no tener conflictos de interés. 


\section{REFERENCIAS BIBLIOGRÁFICAS}

1. Frenk J, Chen L, Bhutta Z, Cohen J, Crisp N, Evans T, et al. Profesionales de la salud para el nuevo siglo: transformando la educación para fortalecer los sistemas de salud en un mundo interdependiente. Rev Peru Med Exp Salud Publica. 2011;28(2):337-41.

2. Organización Panamericana de la Salud; Organización Mundial de la Salud. Salud de los trabajadores en la región de las América. Cuadragésimo Primer Consejo Directivo [Internet]. Ginebra: OPS/OMS; 1999 [citado el 01 de junio de 2014]. Disponible en: http://www.who.int/occupational_ health/regions/en/oeh41 consejo.pdf ?ua $=1$

3. Zevallos L, Pastor R, Moscoso B. Oferta y demanda de médicos especialistas en los establecimientos de salud del Ministerio de Salud: brechas a nivel nacional, por regiones y tipos de especialidad. Rev Peru Med Exp Salud Publica. 2011;28(2):177-85.

4. Perú, Contraloría General de la República. Nota de Prensa $N^{\circ}$ 120-2013-CG/COM: La Contraloría General recomendó identificar y brindar tratamiento médico a pobladores de Madre de Dios afectados por mercurio [Internet]. Lima: Contraloría General de la República; 2013. [citado el 01 de junio de 2014]. Disponible en: http://www.who.int/ occupational_health/regions/en/oeh41consejo.pdf ?ua=1

5. World Health Organization. Training and Education in Occupational Health. Report of a WHO Study Group. Technical Report Series 762 [Internet]. Geneva: WHO; 1988. [citado el 16 de julio de 2014]. Disponible en: http://apps. who.int/iris/bitstream/10665/39382/1/WHO_TRS_762. pdf ?ua $=1$

Correspondencia: Akram Abdul Hernández Vásquez

Dirección: Calle Claudio Gay 2440, Departamento 143, Santiago Centro, Chile

Teléfono: +56953211215

Correo electrónico: akram.hernandez.v@upch.pe

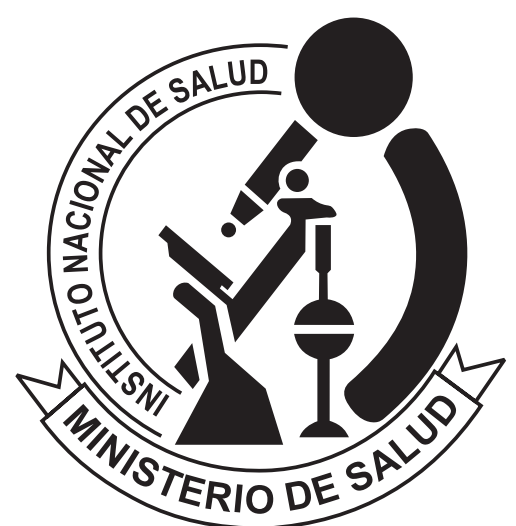

Investigar para proteger la salud

\section{PERCEPCIÓN DE LA FORMACIÓN DURANTE EL RESIDENTADO MÉDICO EN HOSPITALES DEL MINISTERIO DE SALUD DE LIMA, PERÚ}

\section{PERCEPTION OF TRAINING DURING MEDICAL RESIDENCY IN HOSPITALS OF THE MINISTRY OF HEALTH OF LIMA, PERU}

\section{Percy Herrera-Añazco ${ }^{1,5}$, Adrian V. Hernández ${ }^{2,}$, Flor Sánchez-Rivas ${ }^{3,5}$, Christian Arana Maestre ${ }^{4}$}

Sr. Editor. Un reciente informe del Ministerio de salud (MINSA) pone en evidencia la falta de médicos especialistas en Perú (1). Sobre esa base, la Comisión Nacional de Residentado Médico (CONAREME) creó nuevas sedes de residencia o amplió las vacantes en las sedes ya existentes ${ }^{(2)}$. Sin embargo, desde el 2005 no se ha publicado un análisis del proceso de acreditación de sedes docentes para el residentado médico en Perú, a pesar de que en dicha ocasión se reportó que CONAREME no examinó a los programas ya instalados antes del inicio de la evaluación y se les colocó el título de "autorizados", representando estos el $88 \%$ de los programas existentes en ese entonces ${ }^{(3)}$.

El proceso de evaluación de una sede docente es un proceso complejo que debería incluir, entre otras cosas, la percepción de los depositarios del proceso de formación: los residentes, más aun si se plantea ampliar las vacantes de residentado médico sin considerar si las sedes docentes están en capacidad de aceptar nuevos estudiantes brindándoles condiciones mínimas de calidad educativa ${ }^{(4)}$. Con base en ello, realizamos un estudio sobre médicos que cursaban el último mes de entrenamiento de la residencia de medicina interna y especialidades en el Hospital Nacional 2 de Mayo, el Hospital Arzobispo Loayza y el Hospital María Auxiliadora, en mayo de 2014. Se utilizó un cuestionario autoaplicado elaborado en base a evaluaciones previas y consulta de expertos. El cuestionario constó de cinco

\footnotetext{
Hospital Nacional Dos de Mayo. Lima, Perú.

Universidad Peruana de Ciencias Aplicadas. Lima, Perú.

Hospital Nacional Arzobispo Loayza. Lima, Perú.

Hospital María Auxiliadora. Lima, Perú.

Universidad Nacional de Piura. Piura, Perú.

Recibido: 02-07-14 Aprobado: 09-07-14
}

Citar como: Herrera-Añazco P, Hernández AV, Sánchez-Rivas F, Arana Maestre C. Percepción de la formación durante el residentado médico en hospitales del Ministerio de Salud de Lima, Perú. Rev Peru Med Exp Salud Publica. 2014;31(3):606-7. 